\title{
THE CLINICAL PATHOLOGY OF GALL-BLADDER DISEASE
}

\author{
By L. R. JANES, M.A.(Cant.), M.B., B.Chir. \\ (Pathologist, Stephen Ralli Lab., Royal Sussex County Hosp., etc.)
}

\section{Normal and Abnormal Function}

The chief function of the gall-bladder is to act as a reservoir for the bile secreted by the liver during the interdigestive period. This storage of bile is made possible by absorption of water, and the bile is thus concentrated three to ten times. Normally, the main biliary constituents, viz. bile salts, bilirubin, calcium and cholesterol, are not absorbed, but if the gall-bladder is obstructed these substances are absorbed differentially, and ultimately all disappear and are replaced by the mucus secreted by the organ. During the normal process of reduction in bile volume, an equalisation of pressure is effected in the biliary system, and the loss of this function is probably a factor in the dilatation of the ducts that usually occurs after cholecystectomy.

The gall-bladder fills during the fasting state as the sphincter of Oddi is tonically contracted and the liver secretion of bile, although variable, is continuous. Following a meal, the passage of chyme from the stomach into the duodenum causes a relaxation in the tone of Oddi's sphincter and simultaneously the hormone cholecystokinin is liberated from the duodenal mucosa. 'The latter, after absorption into the bloodstream, stimulates the gall-bladder musculature to contract and to empty its contents into the duodenum. Fats, especially egg yolk and cream, are the most effective stimulus to cholecystokinin production, and hence for the emptying of the gallbladder. There is, therefore, a tendency to gall-bladder stasis if fats are absent from the diet and in pathological states, apart from acute cholecystitis, it would appear advisable to include as much fat in the diet as possible without producing pain.

Absorption of the bile salts in the duodenum from the concentrated gall-bladder bile produces a choleretic action on the liver so that liver bile continues to be added to the duodenal contents throughout the digestion of the meal.

Functional disturbances in the normal reciprocal action of relaxation of Oddi's sphincter and gall-bladder evacuation are now well recognised.

Three main types of this disordered motor function (biliary dyskinesia) can thus be distinguished on a physiological basis.

I. The hypermotile type in which rapid emptying of a hypermotile gall-bladder may produce colicky pain.

2. The hypertonic type where the gall-bladder attempts to contract against a spastic sphincter. region.

3. The atonic type with spasm of the sphincter producing chronic pain in the gall-bladder

It may well be that some of the failures following cholecystectomy in the absence of stones are due to a similar disturbance of motor function. Further, some suggest that such abnormalities of evacuation by producing biliary stasis, may be the primary factor in chronic cholecystitis and stone formation. Future experience may well establish the biliary dyskinesias as definite clinical entities.

\section{PATHOLOGY OF GALL-BLADDER DISEASE}

Apart from the rare congenital abnormalities and the uncommon benign and malignant tumours of the biliary apparatus, the overwhelmingly predominant lesion found in the gall-bladder is cholecystitis with or without stone. MacCartey found acute or chronic inflammation in more than 99 per cent of 29,70r surgically removed gall-bladders. Tumours and other pathological conditions were almost always accidental findings at operation for cholecystitis. There is a great difference of opinion as to the common method of bacterial invasion of the gall-bladder. The organ appears to be very resistant to invasion from the bile in spite of the fact that the liver acts as a major bacteriological filter. Similarly, ascending infection from the duodenum via the common bile duct is uncommon except in the presence of achlorhydria and some motor dysfunction of the biliary apparatus. Hurst (I935) favours this route as a common method, and suggests that infection occurs during periods of temporary achlorhydria that may accom- 
pany disturbance of the gastro-intestinal tract. A certain amount of experimental evidence favours hepatogenous infection via the portal vein, and thence to the gall-bladder by the hepatic lymphatics. The local hepatitis commonly found may support this view, although the hepatitis may well follow the cholecystitis. Haematogenous infection via the hepatic artery from a focus in the oral cavity or alimentary tract is favoured by many, and both clinical and experimental work suggests that this method does occur in certain cases at any rate.

Bacteriological studies have given very varied results as to the predominant organism, although nearly all workers agree that organisms are found more frequently in the gall-bladder wall than in the bile, and that they may not be responsible for the primary injury to the organ, but be secondary invaders lighting up an active cholecystitis when stasis or partial obstruction due to stones occurs. Streptococci of non-haemolytic or viridans type, B. coli and staphylococci are the predominant organisms found, but many other bacteria have been isolated on occasion, including $\mathrm{B}$. welchii, members of the typhoid-salmonella group, and $\mathrm{Br}$. abortus. In the more acute forms of infection which may follow acute respiratory or infectious disease or septicaemic states the organism is of high virulence. Mention should be made that apparently chemical or bacterial products, apart from actual organisms, may cause inflammatory changes in the gallbladder. Wolfer (I93I) considers that occasionally pancreatic juice may enter through the common bile and cystic ducts and produce a chemical cholecystitis. Aronsohn and Andrews (I909) have produced an acute cholecystitis in animals by injecting via the cystic duct bile. salts in slightly greater concentration than in gall-bladder bile.

\section{Acute cholecystitis.}

Acute cholecystitis usually arises in a chronically inflamed organ containing stones, one of which may obstruct the cystic duct. Rarely, it occurs as a primary disease in the course of acute infections, disease or septicaemia. In the acute catarrhal forms there is congestion of the organ, but if suppuration develops the mucosa may ulcerate and the enlarged gall-bladder, purplish or blue-green in colour, be distended with purulent bile or pure pus. A peritoneal exudate may be present with adhesions to surrounding structures. The severe forms of infection are usually associated with obstruction of the cystic duct by stone or inflammatory exudate. Intravesical tension rises with increase in fluid content, and should interference with the blood supply follow, gangrene at the fundus or beneath a stone incarcerated in the wall will occur. Perforation into the alimentary tract or peritoneum with the development of peritonitis (often localised by omentum) may follow. Rarely an associated cholangitis may be present with cholecystitis in the absence of stone. Acute cholecystitis due to the typhoid group of organisms may arise during the course of the attack of enteric fever, usually in the third or fourth weeks, or it may occur as an acute suppurative condition years after. More commonly, a low-grade infection may persist for years without symptoms, and a carrier state be present. Whipple, in his review, states that cholecystectomy cures the carrier state in about 70 per cent of cases only.

\section{Chronic cholecystitis.}

In chronic cholecystitis, the pathological lesion varies from the mild catarrhal form with some oedema and round cell infiltration of the mucosa, to complete mucosal destruction and sclerosis of the wall. Sometimes, a proliferation of the epithelium occurs with new gland formation, the condition often called "cholecystitis glandularis proliferans." Stones are frequently present. The gall-bladder may be distended with mucus, but is more often shrunken and thickened.

\section{Cholesterolosis of the gall-bladder.}

It is still obscure how this well-known lipoid infiltration of the mucosa arises. The latest work shows that rarely does the simple lipoid gall-bladder harbour a demonstrable organism, and, indeed, the condition is rare if severe inflammatory or degenerative change is present. Mackey, in his review, concludes that the condition results from a supersaturation of the bile with cholesterol with absorption of the lipoid by the mucosa, and in many cases cholesterol stone formation by crystallisation either on a pigment nucleus or without a nucleus. About one-third of such "strawberry" gall-bladders contain cholesterol rich stones. 
Gallstones.

Autopsies have shown that the incidence of cholelithiasis is high in the adult population, especially in women. Whilst it has to be admitted that the actual mechanism of stone formation has not yet been determined, it would appear certain that this mechanism varies with the type of stone formed.

The pure cholesterol solitaire and the pigment stones of calcium bilirubinate (seen typically in haemolytic jaundice) arise from precipitation in the absence of infection. The common mixed stone consisting of various proportions of cholesterol, bile pigment and calcium, arise in the presence of infection. Calcium carbonate stones are rare, and appear to form only when the cystic duct is completely obstructed, and usually advanced degenerative changes are present in the gall-bladder wall.

Space prevents a detailed discussion of the various factors suggested as causative, but besides infection, stasis and high biliary cholesterol content, it would appear that diminution in the bile acid-cholesterol ratio of the bile, either by a reduction in the bile acid or increase in the cholesterol content, would favour precipitation of cholesterol. Such changes may occur in bile secreted by a liver with dysfunction (Ândrews et al.) (I932), or in altered permeability of the gall-bladder wall in infection or obstruction (Ravdin et al.) (I934). Rous, McMaster and Drury (1923) have shown that changes in the $\mathrm{pH}$ of bile may be important, as a change to the alkaline side tends to precipitate cholesterol and calcium in bile. This may well occur if bacteria are growing in the bile or the wall of the gall-bladder is infected.

Gall-stones have been known to develop in a relatively short time, e.g. within two to three months, and as many as I4,00o have been found in one gall-bladder. Large stones may be formed by agglomeration of smaller ones.

Although many subjects with cholelithiasis do not complain, frequently certain mechanical effects result therefrom producing symptoms. Thus, obstruction of the cystic duct in the absence of infection may lead to absorption of the bile and replacement by the mucus secreted, giving rise to a large mucocoele. If infection is superadded, acute gangrene with perforation may occur. If stones enter the common bile duct, obstructive jaundice generally associated with a shrunken gall-bladder, but with distension of the biliary tree, may result. Suppression of bile flow and replacement by the watery secretion of the ducts ("white bile") may follow. A stone in the ampulla of Vater may lead to chronic or more rarely acute pancreatitis. Calculi may ulcerate through the gall-bladder wall into the duodenum, stomach, small intestine or colon, and intestinal obstruction may result. Biliary fistulae with the pleural cavities or externally (without previous operation) are extremely rare.

\section{Tumours.}

Benign tumours of the gall-bladder are uncommon. Small papillomas consisting of hypertrophied villi usually infiltrated with lipoid are frequent, but true villous papillomata are great rarities. Adenoma, fibromyoma, lipoma and myxoma have been described.

Carcinoma is relatively uncommon (about 3 per cent of all malignant neoplasms), and occurs much more frequently in women. Calculi which are almost always primary are usually present. The commonest form is the scirrhous adeno-carcinoma, but colloid carcinoma is not infrequent. A papillary adeno-carcinoma and squamous carcinoma occur more rarely. The latter presumably arises in the epithelium following squamous metaplasia. These carcinomata are usually highly malignant and quickly invade the liver and surrounding structures, including the common bile duct, and often more distant structures, such as the ovary and the supraclavicular lymph nodes. Secondary carcinoma, largely involving the serous coat, is uncommon.

\section{THE VALUE OF LABORATORY TESTS IN DIAGNOSIS}

The diagnosis of gall-bladder disease rests in the main on the analysis of the patient's symptoms supported by a cholecystogram suggesting a non-functioning organ, or showing calculi formation.

In general, the value of laboratory tests (as is largely that of the physical examination) is chiefly in excluding some of the diseases which enter into the differential diagnosis.

A total and differential white count, routine examination of a catheter (or clean) specimen of urine, and a blood Wassermann reaction should be done on all cases. 
Many cases of acute cholecystitis and some of biliary colic show a slight leucocytosis (I2,000 per c.mm.), but in general the total leucocyte count and Arneth count are of little help. Repeated white cell counts are more useful, for increasing leucocytosis usually accompanies the development of empyema, gangrene, perforation, and cholangitis.

Any changes, such as hypochromia, macrocytosis or spherocytosts, seen in the red cells indicating the presence of anaemia are most important, for anaemia is rarely seen in gall-bladder disease, whether due to inflammation or tumour. Such findings should lead to the exclusion of anaemia due to chronic blood loss from a stomach or duodenal lesion, Addisonian and haemolytic anaemia. There is, of course, a high incidence of biliary stones in haemolytic jaundice, and stones are common in pernicious anaemia. Hyperbilirubinaemia and urobilinuria are characteristic of both diseases. Spherocytosis, reticulocytosis, and typically increased red cell fragility are diagnostic of haemolytic jaundice.

Examination of the urine is of little help in diagnosis, but will exclude acute pyelitis and pyonephrosis, and red cells may be present suggesting renal calculi. All of these urinary tract conditions may simulate gall-bladder disease. Bile in the urine will confirm the scleral or palatal jaundice usually present when the renal threshold for bile is reached.

The Wassermann reaction is practically always positive in hepatic syphilis. The latter, because of the pain, fever, jaundice, and hepatic enlargement is easily confused with subacute cholecystitis. In the crises of tabes dorsalis, the Wassermann reaction may be negative, but 'the presence of the physical signs of this disease should lead to the examination of the cerebrospinal fluid which should include a Lange's gold curve.

Hurst, Dowling (I929) and others have shown that hypochlorhydria and achlorhydria are common in cholecystic disease, but it is now thought that this subacidity represents in most cases the normal depression of gastric acidity found with increasing age. Gastric analysis is therefore of little value in diagnosis, but may help to determine the appropriate treatment of the dyspepsia.

Jaundice in acute cholecystitis is usually due to stone in the common bile duct, but may also be due to cholangitis or hepatitis. Latent jaundice shown by hyperbilirubinaemia with a direct reaction in the $V$ an den Bergh reaction are more common in the acute attacks, but it is rare for this latent jaundice to persist between the attacks except in common duct obstruction-

A single Van den Bergh test, apart from a serum giving an indirect reaction suggesting haemolytic anaemia or pernicious anaemia, has little value in distinguishing between the causes of jaundice. It should be remembered that stone in the biliary ducts is not uncommon in haemolytic anaemia, in which case a direct reaction in the Van den Bergh test may be recorded.

Daily Van den Bergh or Icterus index estimations, however, may be of great value for complete biliary obstruction is rare in common duct stone, and characteristically there are great fluctuations in the serum bilirubin, not always expressed in skin-colour changes. Incomplete obstruction in common duct stone is also shown by the presence of stercobilin in the faeces and urobilin in the urine. It is wise to test faeces chemically for absence of bile. Absence of colour is not reliable,

In common duct obstruction by carcinoma, daily bilirubin estimations show typically a high rising curve; if due to stricture of the common duct, the associated biliary cirrhosis being common, low-fixed curves of serum bilirubin are the rule.

In acutely ill patients with acute cholecystitis or obstructive jaundice, especially if leftsided epigastric pain is present, acute pancreatitis should be suspected and a serum lipase or diastase and urinary diastase estimated.

Much work has been done on liver function tests in biliary disease. Hepatitis is common in these conditions, but, if jaundice is absent, is localised usually to the gall-bladder fossa. The general functions of the liver are therefore usually undisturbed. If jaundice be present, the useful liver function tests are of two types:

(I) those depending on the estimation of substances in the blood excreted by the liver and forced back into the circulation by obstruction of the biliary passages, e.g. plasma cholesterol and serum phosphatase, and

(2) those depending on the estimation of a liver function which are little affected in the early stages of obstructive jaundice, but are seriously disturbed in hepatogenous jaundice. Perhaps the galactose tolerance test (oral or intravenous procedure) which tests the carbohydrate function and the hippuric acid test (oral or intravenous method) which tests the detoxicating function are the two tests most frequently employed. 
There is little doubt that in jaundice of short duration these tests may be of considerable value, but even at this period the obstruction of the biliary passages is often incomplete, so that no alteration may occur in the blood, and further many cases of hepatogenous jaundice show an obstructive element. Obstructive jaundice, if persistent, sooner or later produces grave liver damage, and this is revealed by tests for liver function. It will be seen that these tests may be of great value in an individual case, but are unlikely to help in the more chronic forms of jaundice.

\section{Duodenal Intubation.}

The Lyon-Meltzer test consists of an examination of the bile obtained by duodenal intubation after the installation of magnesium sulphate or some other biliary stimulant. This test is frequently employed in America and on the Continent as an aid to the diagnosis of biliary disease, but in Great Britain reliance is placed on cholecystography for the most part, and it has never attained a widespread popularity. It is used by some in this country as an aid to the diagnosis of catarrhal cholecystitis. The details of the technique of the test may be found in any of the textbooks on clinical pathology or in Lyon's treatise.

The darkly coloured bile, so-called "B" bile, obtained after magnesium sulphate stimulation of the duodenum may be assumed to have come from the gall-bladder, and its dark colour indicates that the gall-bladder can concentrate liver bile. If repeated intubation fails to yield " $\mathrm{B}$ " bile and radiological evidence shows the tube to be in the duodenum, it is considered that disease of the gall-bladder is present. This " $\mathrm{B}$ " bile fraction is measured and examined for cholesterol crystals, calcium bilirubinate pigment, mucus, epithelial cells, pus and bacteria. In surveying the literature, there is a remarkable divergence of opinion as to the significance placed upon the presence of these various substances. Many consider the presence of cholesterol crystals and calcium bilirubinate pigment pathognomonic of calculus formation, others say that it indicates disturbed biliary chemistry, but not necessarily stone. The presence of cellular and bacterial elements indicate to many an inflammatory lesion. Others, remarking on the lack of agreement between cultures obtained at operation from the gall-bladder and the contained bile itself, with that obtained at duodenal drainage, suggest that most of the bacteria cultured from the latter procedure are contaminants either from the oral cavity or from the duodenum in the presence of achlorhydria. However, typhoid carriers have frequently been detected by the method. Fidler, Innes and Davidson (I94I) found that degenerate epithelial cells present in the bile may be produced by the local irritant action of magnesium sulphate on the duodenal mucosa. The absence of formed elements does not exclude gall-bladder pathology. Exponents of the method appear to agree that the procedure is time consuming, requires a co-operative patient and a highly-skilled worker, exercising meticulous technique.

\section{TESTS TO DETERMINE OPERATIVE RISKS}

It is now recognised that the haemorrhagic tendency in the jaundiced patient is due to insufficient prothrombin in the blood. The normal synthesis of prothrombin by the liver depends on an adequate absorption of the fat soluble vitamin $\mathrm{K}$ from the gut, and in the absence of bile salts this tends to be defective. A diet deficient in vitamin $K$ will produce a similar reduction in blood prothrombin, and it should be remembered that a patient with long-standing gallbladder disease, in attempting to avoid foods which produce dyspepsia, may suffer from malnutrition. Further, if liver damage is severe, prothrombin formation may be markedly reduced and may not improve with vitamin $\mathrm{K}$ therapy.

It is therefore essential to estimate the prothrombin time (by Quick's method or one of its modifications) in all cases of jaundice, and it would be wiser to do so in those without jaundice who have lost much weight with biliary disease. If the prothrombin time is less than 85 per cent of normal, vitamin $\mathrm{K}$ therapy should be given until the prothrombin time is above this level. In the presence of grave liver damage, this level will not be reached, and if operation is attempted blood transfusion must be given. Cohn of Harvard has recently separated prothrombin from human plasma, and exhibition of this, when available, would appear to be the ideal method of preventing haemorrhage in such cases (Ravdin, I942).

Another operative risk which pre-operative therapy may often overcome is reduced hepatic efficiency. Liver damage is naturally most frequently found in chronic biliary obstruction, but it may be present in those, without jaundice, who give a long history of gall-bladder infection especially if obese. Other individuals appear either to be born with inferior livers or acquire 
subnormal hepatic function in the process of living (Heyd (I937), Hurst (I938)). Recent work has suggested that pre-operative diets containing protein rich in sulphur amino-acids (such as casein) and carbohydrates would appear to be of value in protecting the liver from the further injury inevitably inflicted by operation. The great difficulty, of course, is that there is no known test for liver function that is universally applicable to determine reduced hepatic efficiency. This is not surprising when the great number of functions the liver performs is considered. Further, in various conditions and under different conditions of nutrition, these functions are not affected to the same extent by noxious agents. Nevertheless, from a clinical point of view, results obtained by some of the modern tests, taken in conjunction with the clinical appearances, are valuable.

It is not possible here to discuss or to attempt to evaluate the various tests employed, but the performance of several different tests is probably the ideal. However, for clinical purposes, provided renal function (shown by blood urea) is normal, the hippuric acid synthesis test devised by Quick has been found to be satisfactory in evaluating surgical risk in either the jaundiced or non-jaundiced patient. The test depends on the ability of the liver to conjugate benzoic acid and amino-acetic acid. In the oral method 6 gms. of sodium benzoate are given in water by mouth, and the hippuric acid estimated gravimetrically in the urine passed in the next four hours. Values of $2 \cdot 6$ to $3 \cdot 3$ gms. (as benzoic acid) are considered normal. Rosenberg et al. (I94I) suggests that in slight degrees of hepatic damage an increased excretion may be obtained. It has been found that if less than I. 5 gms. of benzoic acid is excreted, the prognosis is bad, and few patients survive operation.

The value of repeated blood bilirubin estimations has been mentioned earlier in this paper.

Finally, in spite of the limitations of liver function tests, for none are infallible, present experience does show that where they are employed as a routine they have yielded results that have proved well worth while. In my view, the time is not far distant when tests for liver function in biliary disease will be considered by operative surgeons as essential as renal efficiency tests in renal disease.

\title{
REFERENCES
}

WOLFER, J. A., "The role of the pancreatic juice in the production of gall-bladder disease," Surg., Gynec. and Obst., 53, 433-447, I93 ARONSOHN, H. G., and ANDREWS, E., Experimental cholecystitis, Jena, G. Fischer, I909.

WHIPPLE, A. O., "'The surgical treatment of typhoid carriers," Ann. Surg., 90, 63I-642, 1929.

MACKEY, W. A., Brit. J. Surg., 24, 570.

ANDREWS, E., SCHOENHEIMER, R., and HRDINA, L., "Etiology of gallstones," Arch. Surg., 25, 796-8ro, r932.

RAVDIN, I.S., RIEGEL, C., JOHNSTON, C. G., and MORRISON, P. J., "Studies in biliary tract disease,"J.A.M.A., 103, I5O4, I934. ROUS, P., McMASTER, P. D., and DRURY, D. R., "Genesis of gallstones in the dog," Proc. Soc. Exper. Biol. and Med., 20, $3 \mathrm{I} 5$. I923.

HURST, A. F., "Disorders of the gall bladder," Guy's Hosp. Gaz., 49, 2 16, 1935.

DOWLING, G.' A., "Dyspepsia due to gall-bladder disease," J.A.M.A., 92, 7, I929.

LYON, B. B. V., Non-surgical drainage of gall tract, Philadelphia, Lea and Febiger, 1923

FIDLER, A., INNES, J., DAVIDSON, J. S. P., B.M.J., 2, 866, I94I.

RAVDIN, I. S., "A consideration of some problems of biliary tract disease," Proc. Inst. Med., Chicago, 14, 173, 19+2.

HEYD, C. G.,, "Liver deaths and the complications of gall-bladder surgery, South Surgeon, 6, I83, 1937.

HURST, A. F., B.M.J., 1, 661, I938.

QUICK, A. J., "The synthesis of hippuric acid: A new test of liver function," Am. J.M. Sc., 185,630, I933.

ROSENBERG, D. H., SOSKIN, S., Am. J. Dig. Diseases, 8, 425, I94I.

\section{SOME ASPECTS OF THE CLINICAL DIAGNOSIS OF ACUTE AFFECTION OF THE GALL BLADDER AND BILE DUCTS}

\author{
By ALEXANDER CRUICKSHANK, M.A., M.B., F.R.C.S. \\ (Assist. Surg., Prince of Wales Hosp., Tottenham, Queen's Hosp. for Children; Surg., Acton, \\ Teddington, Richmond Hosps., etc.; Surg. to E.M.S.)
}

It has been established by exhaustive researches into the medical history of disease of the $\stackrel{\mathscr{Q}}{?}$ biliary apparatus that the first mention of gall-stones and acute liver disease was made by a 0 Greek physician in the fifth century. His writings were published much later, in fact, in the 